\title{
Magnetic tilting and emergent Majorana spin connection in topological superconductors
}

DOI:

10.1103/PhysRevB.98.094515

\section{Document Version}

Final published version

Link to publication record in Manchester Research Explorer

\section{Citation for published version (APA):}

Chirolli, L., \& Guinea, F. (2018). Magnetic tilting and emergent Majorana spin connection in topological superconductors. Physical Review B, 98(9), [094515]. https://doi.org/10.1103/PhysRevB.98.094515

\section{Published in:}

Physical Review B

\section{Citing this paper}

Please note that where the full-text provided on Manchester Research Explorer is the Author Accepted Manuscript or Proof version this may differ from the final Published version. If citing, it is advised that you check and use the publisher's definitive version.

\section{General rights}

Copyright and moral rights for the publications made accessible in the Research Explorer are retained by the authors and/or other copyright owners and it is a condition of accessing publications that users recognise and abide by the legal requirements associated with these rights.

\section{Takedown policy}

If you believe that this document breaches copyright please refer to the University of Manchester's Takedown Procedures [http://man.ac.uk/04Y6Bo] or contact uml.scholarlycommunications@manchester.ac.uk providing relevant details, so we can investigate your claim.

\section{OPEN ACCESS}




\title{
Magnetic tilting and emergent Majorana spin connection in topological superconductors
}

\author{
Luca Chirolli ${ }^{1}$ and Francisco Guinea ${ }^{1,2}$ \\ ${ }^{1}$ IMDEA-Nanoscience, Calle de Faraday 9, E-28049 Madrid, Spain \\ ${ }^{2}$ School of Physics and Astronomy, University of Manchester, Manchester M13 9PY, United Kingdom
}

(Received 9 March 2018; revised manuscript received 24 July 2018; published 18 September 2018)

\begin{abstract}
Due to the charge-neutral and localized nature of surface Majorana modes, detection schemes usually rely on local spectroscopy or interference through the Josephson effect. Here, we theoretically study the magnetic response of a two-dimensional cone of Majorana fermions localized at the surface of class-DIII topological superconductors. For a field parallel to the surface the Zeeman term vanishes, and the orbital term induces a Doppler shift of the Andreev levels, resulting in a tilting of the surface Majorana cone. For fields larger than a critical threshold field $H^{*}$ the system undergoes a transition from a type-I to type-II Dirac-Majorana cone. In a spherical geometry the surface curvature leads to the emergence of the Majorana spin connection in the tilting term via an interplay between orbital and Zeeman terms that generates a finite nontrivial coupling between negative- and positive-energy states. Majorana modes are thus expected to show a finite response to the applied field, which acquires a universal character in finite geometries and opens the way to detection of Majorana modes via time-dependent magnetic fields.
\end{abstract}

DOI: 10.1103/PhysRevB.98.094515

\section{INTRODUCTION}

Majorana particles are charge-neutral fermions, and as such they do not couple to the electromagnetic field [1]. In solid-state systems [2] they are formed as the zero-energy equal superposition of particle and hole states, localized at a vortex core [3] or at the boundary of topological superconductors [4,5]. Majorana fermions constitute a particular class of topologically protected surface states appearing at the boundary of topological states of matter [6-10], and they represent one of the basic resources in topological quantum computation [11,12]. Majorana states localized at the surface of two-dimensional (2D) and three-dimensional (3D) topological superconductors form modes that fill the entire gap [13-15]. In class-DIII topological superconductors in $D=3$ dimensions [16-20], Majorana modes form a time-reversalinvariant (TRI) Dirac cone in the basis of Majorana Kramers partners. A novel class of material based on doped $\mathrm{Bi}_{2} \mathrm{Se}_{3}$ topological insulators (TIs) [6,9,21-24] has been suggested as a candidate that may realize odd-parity, TRI topological superconductivity [25-32]. Due to their charge neutrality and localized character, most of the theoretical proposals and experimental efforts to detect Majorana states have focused on local spectroscopy, the Josephson effect, and interferometry as proof of their existence $[33,34]$. In addition, surface Majorana modes are expected to produce a strong anisotropy in the spin susceptibility [35].

In this work we study the coupling of a $2 \mathrm{D}$ cone of Majorana fermions localized at the surface of a class-DIII topological superconductor to an externally applied magnetic field. Meissner screening fixes the relevant field orientation to lie on the surface, and the system responds with a supercurrent. The latter gives rise to a Doppler shift of the energy levels, which results in a tilting of the Majorana cone along the supercurrent direction. Upon increasing the external field a threshold field $H^{*}$ is reached for which the dispersion becomes flat along the tilting direction and the Majorana cone experiences a structural change from a type-I to type-II Dirac-Majorana cone [36,37]. $H^{*}$ is upper bounded by the thermodynamic critical fields $H_{c}$, and for field smaller than $H^{*}$ the system is not expected to respond to the field. For highly doped small-band-gap Dirac insulators hosting odd-parity superconductivity, such as the $A_{1 u}$ phase predicted in $\mathrm{Bi}_{2} \mathrm{Se}_{3}$ [25], $H^{*}$ can lie within the Meissner phase, thus making accessible the type-II Dirac cone regime.

In systems characterized by a finite surface curvature the triplet nature of the pairing leads to an additional nontrivial coupling of geometric origin to the external field. The tilting term, involving only the momentum operator on a flat surface, necessarily leads to the emergence of the Majorana spin connection on curved space and adds to the Zeeman term, both involving the spin density operator perpendicular to the surface. The external field thus generates a finite coupling between occupied and empty states, and a Majorana-photon coupling thus appears on curved surfaces, allowing detection of surface Majorana states by application of time-dependent magnetic field. Our findings acquire a universal character in finite geometries characterized by a nonzero curvature and open the way to detection and manipulation of Majorana states via electromagnetic fields.

\section{THE SYSTEM}

We start our analysis by considering a generic example of class-DIII topological superconductors, the Balian-Werthamer (BW) state [38], which describes time-reversal-invariant triplet pairing of the form $(\mathbf{p} \cdot \mathbf{s}) i s_{y}$. The mean-field Hamiltonian in the Nambu basis $\psi_{\mathbf{k}}=\left(c_{\mathbf{k} \uparrow}, c_{\mathbf{k}, \downarrow}, c_{-\mathbf{k}, \downarrow}^{\dagger},-c_{-\mathbf{k}, \uparrow}^{\dagger}\right)^{T}$, with $c_{\mathbf{k}, s}$ being a 
fermionic state of momentum $\mathbf{k}$ and spin $s$, takes the form $\mathcal{H}=\frac{1}{2} \sum_{\mathbf{k}} \psi_{\mathbf{k}}^{\dagger} \mathcal{H}_{\mathrm{BW}}(\mathbf{k}) \psi_{\mathbf{k}}$, where the Bogoliubov-de Gennes (BdG) Hamiltonian is given by

$$
\mathcal{H}_{\mathrm{BW}}=\tau_{z}\left(\frac{\mathbf{p}^{2}}{2 m}-\epsilon_{F}\right)+\frac{\Delta}{\hbar k_{F}} \tau_{x} \mathbf{p} \cdot \mathbf{s},
$$

where $m$ is the effective mass of the electrons, $\epsilon_{F}$ is the Fermi energy, $k_{F}=\sqrt{2 m \epsilon_{F}} / \hbar$ is the Fermi momentum, $\mathbf{s}$ is the vector of spin Pauli matrices, $\tau_{i}$ are Pauli matrices in the particle-hole Nambu space, and $\Delta$ is the mean-field value of the pseudoscalar order parameter describing the $\mathrm{BW}$ state. The BdG Hamiltonian (1) is time reversal invariant, with $\mathcal{T}=i s_{y} \hat{K}$ and $\hat{K}$ being complex conjugation, and it has a full bulk gap of size $\Delta$ on the Fermi surface.

A surface Majorana-Dirac cone is found by studying the subgap spectrum of the confined problem. By confining the system in the region $z>0$, a zero-energy Majorana Kramers pair is found at $k_{x}=k_{y}=0$. Introducing the coherence length of the superconductor $\xi=\hbar v_{F} / \Delta$ (with $v_{F}=\hbar k_{F} / m$ ), the un-normalized wave function in the limit $k_{F} \xi \gg 1$ is given by

$$
\phi_{s}(z)=|s\rangle\left(|+\rangle_{\tau}+i s|-\rangle_{\tau}\right) e^{-z / \xi} \sin \left(k_{F} z\right),
$$

where $\tau_{z}| \pm\rangle_{\tau}= \pm| \pm\rangle_{\tau}, s_{z}|s\rangle=s|s\rangle$. For $\mathbf{k} \neq 0$ we project the Hamiltonian (1) onto the surface states $\left|\phi_{s}\right\rangle$ and obtain an effective surface Hamiltonian valid for energies below the gap

$$
\mathcal{H}_{\mathbf{k}}^{0}=\hbar v_{\Delta}\left(k_{x} s_{y}-k_{y} s_{x}\right),
$$

with $v_{\Delta}=\Delta / \hbar k_{F}$, which represents a massless DiracMajorana Hamiltonian describing the surface physics.

\section{COUPLING TO A MAGNETIC FIELD}

The magnetic field is introduced in Eq. (1) by minimal coupling substitution, $\mathbf{p} \rightarrow \mathbf{p}+e \mathbf{A} \tau_{z}$ in the kinetic energy, and the gap acquires a dependence on the position. For weak magnetic field in the Meissner regime the vector potential and the gap change over distances on the order of the penetration depth $\lambda$ and the electromagnetic properties of the pseudoscalar order parameter $\Delta$ are similar to those of a scalar $s$-wave order parameter [38-40]. We then assume a constant hard gap that is not affected by the magnetic field. Neglecting the diamagnetic $\mathbf{A}^{2}$ term, the coupling to the magnetic field reads

$$
\mathcal{H}_{B}=\frac{e}{2 m}(\mathbf{p} \cdot \mathbf{A}+\mathbf{A} \cdot \mathbf{p})+\frac{1}{2} g \mu_{B} \mathbf{S} \cdot \mathbf{B}
$$

for both particles and holes, where the second term describes Zeeman coupling to the total induction field $\mathbf{B}=\boldsymbol{\nabla} \times \mathbf{A}$, with $g$ being the electron $g$ factor and $\mu_{B}$ being the Bohr magneton. The BdG Hamiltonian transforms under particlehole $(\mathrm{PH})$ symmetry as $U_{\mathcal{C}}^{\dagger} \mathcal{H}^{*}(-\mathbf{k}, H) U_{\mathcal{C}}=-\mathcal{H}(\mathbf{k}, H)$, with $U_{\mathcal{C}}=s_{y} \tau_{y}$. Consequently, the spectrum has the following symmetry: $\epsilon^{n}(\mathbf{k}, H)=-\epsilon^{n}(-\mathbf{k}, H)$.

When an external field $H$ is applied to the system, Meissner screening forces the external field to lie in the plane defined by the surface of the system. The Zeeman term involves then only in-plane spin components, whose matrix elements on the states (2) are zero. In the Landau gauge the vector potential corresponding to the bulk screened field reads $\mathbf{A}=\left(0, H \lambda e^{-z / \lambda}, 0\right)$, with the field $\mathbf{B}$ pointing about the $x$ direction. Having no left structure in spin and particle-hole

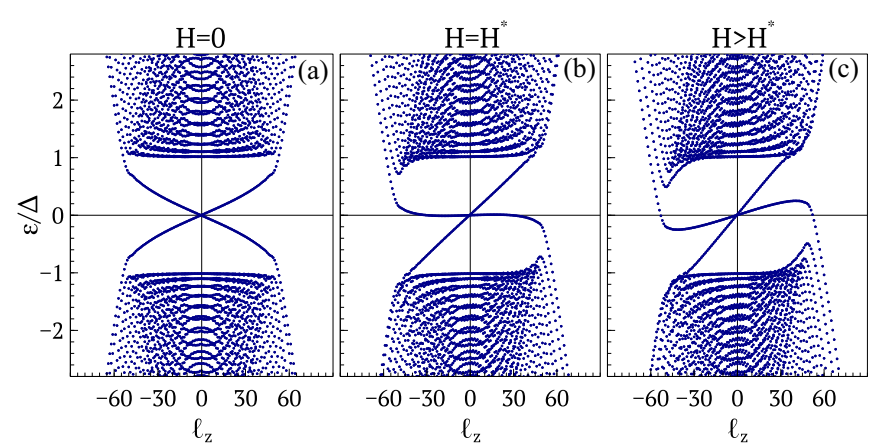

FIG. 1. Evolution with the field of the spectrum on a cylindrical geometry for the case $k_{z}=0$ versus angular momentum $\ell_{z}=j_{z}-$ 1/2. The applied field is $H=0$ in (a), $H=H^{*}$ in (b), and $H>$ $H^{*}$ in (c), and it exponentially decays towards the center of the cylinder on a scale $\lambda=0.06 R$. PH symmetry requires that $\epsilon_{\ell_{z}}^{n}(H)=$ $-\epsilon_{-\ell_{z}-1}^{n}(H)$.

space, the projection onto the Majorana cone of $\mathcal{H}_{B}$ can amount to only a diagonal momentum-dependent term,

$$
\mathcal{H}_{\mathbf{k}}=\hbar v_{\Delta}\left(k_{x} s_{y}-k_{y} s_{x}\right)+\hbar v_{H} k_{y},
$$

where the field-induced velocity is given by $v_{H}=e\left\langle A_{y}\right\rangle / m \simeq$ $e A_{y}(0) / m=e H \lambda / m$, in the limit $k_{F} \xi \gg 1$, and $\langle\cdots\rangle$ stands for the expectation value on the Majorana wave function (2). This term describes a Doppler shift of the energy of the Majorana modes due to the superfluid velocity generated by the field and accounts for the Galilean transformation of the energy levels from the comoving to the laboratory frame [41]. The result is a tilting of the bands described by the energy dispersion $\epsilon_{\mathbf{k}, \pm}=\hbar v_{H} k_{y} \pm \hbar v_{\Delta} k$. Upon increasing the field the tilting becomes more and more pronounced, until a threshold field is reached, where one of the two energy branches becomes flat at the Landau critical velocity $v_{H}=v_{\Delta}$,

$$
H^{*}=\eta \frac{\Phi_{0}}{\pi \xi \lambda}=\eta H_{c}
$$

In the second equality we express the threshold field in terms of the thermodynamic critical field $H_{c}=\Phi_{0} /(2 \pi \xi \lambda)$ [41]. For a type-II superconductor $H_{c}$ lies between the lower and upper critical fields, $H_{c 1}<H_{c}<H_{c 2}$, with $H_{c 1}=$ $\Phi_{0} /\left(4 \pi \lambda^{2}\right) \ln (\lambda / \xi)$ and $H_{c 2}=\Phi_{0} /\left(2 \pi \xi^{2}\right)$, respectively [41]. At $H=H^{*}$ the Majorana cone becomes flat along the line $k_{x}=0$, and a transition from type I to an overtilted typeII Dirac-Majorana cone takes place that is characterized by a formally diverging number of states at zero energy. In Fig. 1 we plot the band structure of the Hamiltonian (7) in a cylindrical geometry with a screened field. From left to right the field is increased from $H=0$ to $H>H^{*}$, and the tilting of the bands is evident, thus confirming the prediction of the low-energy model. The parameter $\eta$ in Eq. (6) is the ratio of the actual Majorana velocity $v_{\Delta}$ and $\Delta / \hbar k_{F}$, and it captures the discrepancy between the actual Hamiltonian and the BW state (1) $(\eta=1$ for the BW state).

\section{A. Odd-parity superconductor}

A relevant example is represented by odd-parity superconductors realized in doped TIs, like $\mathrm{Bi}_{2} \mathrm{Se}_{3}$, or small-band-gap 
doped Dirac insulators. These systems are described by the BdG Hamiltonian [25]

$$
\mathcal{H}=\tau_{z}\left[E_{G} \sigma_{x}+v \sigma_{z}\left(k_{x} s_{y}-k_{y} s_{x}\right)+v_{z} k_{z} \sigma_{y}\right]+\Delta \tau_{x} \sigma_{y} s_{z},
$$

where $\sigma_{i}$ are Pauli matrices spanning the subspace of two orbitals with $p_{z}$-like symmetry, $E_{G}$ is the insulating band gap, and $v$ is the Dirac velocity. The BW Hamiltonian (1) can be seen as the low-energy limit of the Hamiltonian (7) and can be obtained upon projection on the conduction band. Introducing Euclidean Dirac matrices, $\gamma^{\mu}=\left(\gamma^{0}, \gamma^{1}, \gamma^{2}, \gamma^{3}\right)=$ $\left(\sigma_{x},-\sigma_{y} s_{y}, \sigma_{y} s_{x}, \sigma_{z}\right)$ and $\gamma^{5}=\sigma_{y} s_{z}$, and assuming isotropic velocity $v$, minimal coupling substitution $\mathbf{p} \rightarrow \mathbf{p}+\tau_{z} \mathbf{A}$ produces the coupling term $\mathcal{H}_{\mathbf{A}}=-i e v \gamma^{0} \gamma^{i} A_{i}$. Defining $\mathcal{H}_{0}=$ $\tau_{z}\left(E_{G} \gamma^{0}-\mu\right), \mathcal{H}_{\mathbf{p}}=-i v \gamma^{0} \gamma^{i} p_{i} \tau_{z}$, and $\mathcal{H}_{\Delta}=\Delta \tau_{x} \gamma^{5}$, the projected coupling is given by

$$
\tilde{\mathcal{H}}=\mathcal{H}_{\mathrm{BW}}+\mathcal{H}_{\mathbf{p} \cdot \mathbf{A}}+\mathcal{H}_{Z},
$$

where $\mathcal{H}_{\mathrm{BW}}=\left\langle\psi_{c}\left|\mathcal{H}_{0}-\mathcal{H}_{\mathbf{p}} \mathcal{H}_{0}^{-1} \mathcal{H}_{\mathbf{p}}-\mathcal{H}_{\Delta} \mathcal{H}_{0}^{-1} \mathcal{H}_{\mathbf{p}}\right| \psi_{c}\right\rangle$ is the BW state Hamiltonian (1), with $\left|\psi_{c}\right\rangle\left\langle\psi_{c}\right|$ being the conduction band projector, $m=\left(\mu+E_{G}\right) / 2 v^{2}$ and $k_{F}=\left(\mu+E_{G}\right) / 2 v$, $\mathcal{H}_{\mathbf{p} \cdot \mathbf{A}}$ is given by the first term in Eq. (4) in a gauge for which $\boldsymbol{\nabla} \cdot \mathbf{A}=0, \mathcal{H}_{Z}=g^{*} \mu_{B} s_{i} B_{i}$ is the Zeeman term with the effective $g$ factor $g^{*}=m_{\mathrm{e}} / m+g_{\mathrm{Bi}_{2} \mathrm{Se}_{3}} / 2$, with $m_{\mathrm{e}}$ being the bare electron mass, and we have included the material $g$ factor. Importantly, no coupling between the gap matrix and the vector potential arises at second order, $\left\langle\psi_{c}\left|\mathcal{H}_{\mathbf{A}} \mathcal{H}_{0}^{-1} \mathcal{H}_{\Delta}\right| \psi_{c}\right\rangle=$ 0 . The velocity of the Majorana modes derived from Eq. (7) is $v_{\Delta} \simeq v E_{G} \Delta / \mu^{2}$ [42]. Upon identifying $m=\mu / 2 v^{2}$, one has $\eta=E_{G} / \mu$. Thus, by increasing the chemical potential $\mu$ it is possible to shift $H^{*}$ in the Meissner regime.

In an upcoming work [43] we will show that for fields larger than the threshold field, $H>H^{*}$, the surface Majorana modes carry a finite current that contributes to Meissner screening and gives rise to an additional surface diamagnetic moment that can be detected in orbital magnetic susceptibility measurements. The analysis so far presented fully makes sense for weak $H^{*}(\eta \ll 1)$ in the Meissner phase, where the bulk completely expels the external field. For $\eta \sim 1$ vortices start to enter the system before $H^{*}$ is actually reached. In this case, the field can be assumed to be constant, $A_{y}=-H z$, and the tilting field shifts to $H_{c 2}=\Phi_{0} /\left(\pi \xi^{2}\right)$, for which superconductivity is completely destroyed. In addition, the application of high fields may make favorable the condensation of other possible multicomponent superconducting order parameters [44] that due to the unconventional character of the pairing are expected to affect the magnetic phase diagram, and the entire analysis ceases to be valid.

\section{CURVATURE EFFECTS}

We now study the modification of the Majorana cone in curved, finite-size geometries. For simplicity, we will set $\eta=$ 1 in the following. In cylindrical and spherical geometries it is possible to choose the self-consistent vector potential to have only a nonzero azimuthal component $\mathbf{A}=\hat{\boldsymbol{\phi}} A_{\phi}(r)$, with $\boldsymbol{\nabla}$. $\mathbf{A}=0$. The coupling to the magnetic field can be written as

$$
\mathcal{H}_{B}=\frac{e}{m} A_{\phi}(\mathbf{r}) p_{\phi}+g^{*} \mu_{B} \mathbf{B}(\mathbf{r}) \cdot \mathbf{s} .
$$

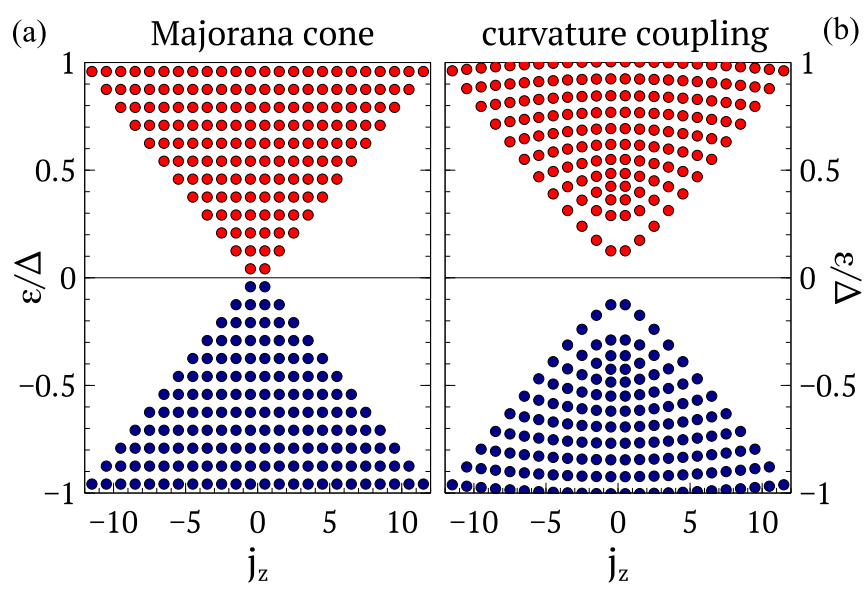

FIG. 2. Sketch of the surface Andreev states of the BW states in a spherical geometry. (a) Surface Majorana cone without magnetic field and (b) the effect of the matrix elements (13) on the spectrum. Large values of the external field have been chosen to highlight the effect.

In both cylindrical and spherical geometries the $z$ component of the total angular momentum $\hat{J}_{z}=\hat{L}_{z}+\hbar s_{z} / 2$, with $\hat{L}_{z}=-i \hbar \partial_{\phi}$ being the $z$ component of the orbital angular momentum $\hat{\mathbf{L}}$, is a constant of motion, and the spectrum can be labeled by $j_{z}$. On the cylinder we find that the surface Hamiltonian is given by the counterpart of Eq. (5) for the surface of the cylinder (results not shown).

On a sphere of radius $R, \mathcal{H}$ commutes with $\hat{\mathbf{J}}^{2}, \hat{J}_{z}$, and $\mathcal{C}=\tau_{z}(\mathbf{s} \cdot \hat{\mathbf{L}}+\hbar)$, with eigenvalues $j(j+1) \hbar^{2}, j_{z} \hbar$, and $\kappa= \pm(j+1 / 2) \hbar$. Thanks to $\{\mathcal{C}, \Theta\}=0$, with $\Theta=\tau_{y} \mathcal{T}$ the particle-hole symmetry operator, the sign of $\kappa$ can be chosen to label the sign of the eigenenergies. In turn, $\left[\mathcal{C}, s_{z}\right] \neq 0$ implies that $s_{z}$ can generate finite matrix elements between the positive- and negative-energy states. Writing $p_{\phi}=\hbar\left(j_{z}-\right.$ $\left.s_{z} / 2\right) /(r \sin \theta)$ and $A_{\phi}(\mathbf{r})=A(r) \sin \theta$, for large $j_{z}$ the spectrum is given by

$$
\epsilon_{j, j_{z}}^{ \pm}= \pm \frac{\Delta}{k_{F} R}(j+1 / 2)+j_{z} \frac{\hbar e \lambda H}{m R}, \quad\left|j_{z}\right| \gg 1 / 2 .
$$

In the absence of the external field every state labeled by $j$ is $2 j+1$ degenerate, corresponding to the values of $j_{z}$, with $\left|j_{z}\right| \leqslant j$ [see Fig. 2(a)]. As for the planar and cylindrical geometries the field produces a tilting of the spectrum, and a threshold field $H^{*}$ arises, whose value agrees well with Eq. (6) (see Appendix C). For small $j_{z}$ the role of the spin becomes important, and both the orbital and Zeeman terms act as relevant perturbations to the Majorana modes. The surface contribution of the Zeeman term reads

$$
\mathcal{H}_{Z}=g^{*} \mu_{B} \frac{3 H}{2}\left[\sin \theta \hat{\boldsymbol{\theta}}-\frac{\lambda}{2 R} \cos \theta \hat{\mathbf{r}}\right] \cdot \mathbf{s} .
$$

The polar term acts in plane, and its matrix elements are negligible (see Sec. C2), so that the relevant perturbation provided by the magnetic field reads

$$
\mathcal{H}_{B}=-\mu_{B} \frac{A(r)}{r}\left(s_{z}-2 g^{*} \cos \theta \hat{\mathbf{r}} \cdot \mathbf{s}\right) .
$$

This term is even under parity, so that it generates finite matrix elements between states that differ by an odd number of an- 
gular momentum quanta. In particular, it generates matrix elements between states of positive energy and quantum numbers $\left(j, j_{z}\right)$ and states of negative energies and quantum numbers $\left(j \pm 1, j_{z}\right)$. A partial cancellation takes place between the orbital term and part of the Zeeman term, and the net matrix elements read (see Sec. C 3)

$$
\left\langle\Psi_{j, j_{z}}^{+}\left|\mathcal{H}_{B}\right| \Psi_{j \pm 1, j_{z}}^{-}\right\rangle=\frac{\tilde{g} \mu_{B} A(R)}{R} \frac{\sqrt{(j+1 / 2 \pm 1 / 2)^{2}-j_{z}^{2}}}{j+1 / 2 \pm 1 / 2}
$$

with $A(R)=3 H \lambda / 2$ on the sphere surface and $\tilde{g}=g^{*}-1 / 2$. Analogously, the Zeeman term, whose relevant component is $s_{z}$, has similar finite matrix elements and couples to the Majorana cone. It becomes clear that the orbital magnetic field adds to the Zeeman term and acts as a relevant perturbation that opens a gap in the Majorana cone. The resulting spectrum without the tilting term is shown in Fig. 2(b). The magnetic field increases the splitting between positive- and negativeenergy states as a result of a nontrivial coupling.

\section{A. Majorana spin connection}

The matrix elements (13) hide a profound geometric origin. The Majorana modes in the absence of the field satisfy a 2D Dirac equation, which on a planar metric is given by Eq. (3). Introducing Dirac matrices $\gamma^{i} \equiv\left(\gamma^{0}, \gamma^{1}, \gamma^{2}\right)=$ $\left(-i s_{z}, s_{y},-s_{x}\right)$, the two-component Majorana spinor $\psi$ satisfies the Dirac equation on a flat Minkowski space $\gamma^{i} \partial_{i} \psi=0$. Parallel transport on the surface of a sphere dictates the way the derivative $\partial_{i}$ becomes replaced by the covariant derivative $D_{\mu}=\partial_{\mu}+\Gamma_{\mu}$. The spin connection, related to the intrinsic curvature of the metric, necessarily emerges at the boundaries of a topological superconductor, and the Majorana spinor satisfies the Dirac equation on the curved space-time $S^{2} \times R$ [45-47],

$$
\gamma^{\mu}\left(\partial_{\mu}+\Gamma_{\mu}\right) \psi=0
$$

where $\gamma^{\mu}=\gamma^{i} e_{i}^{\mu}$ are rotated Dirac matrices satisfying $\left\{\gamma^{\mu}, \gamma^{\nu}\right\}=2 g^{\mu \nu}$, where the curved metric $g_{\mu \nu}=\eta_{i j} e_{\mu}^{i} e^{j}$ replaces the Minkowski metric $\eta_{i j}$ via the tetrads $e^{i}{ }_{\mu} \equiv$ $\partial x^{i} / \partial x^{\mu}$. The spin connection $\Gamma_{\mu}$ is given by $\frac{i}{2} \Gamma_{\mu}^{i}{ }^{j} \Sigma_{i j}$, where $\Sigma_{i j}=\frac{i}{2}\left[\gamma_{i}, \gamma_{j}\right]$ are the generators of the spinorial representation of the Lorentz group and the connection coefficients are given by $\Gamma_{\mu}^{i}{ }_{\mu}^{j}=e^{i}{ }_{\nu} \nabla_{\mu} e^{j v}$. On the surface of the sphere the metric takes the form $g_{\mu \nu}=$ $\operatorname{diag}\left(-1, R^{2}, R^{2} \sin ^{2} \theta\right)$, and by reading off the tetrads the spin connection is found to be

$$
\Gamma_{\mu}=-\frac{i}{2} s_{3} \cos \theta \delta_{2 \mu}
$$

The resulting Dirac Hamiltonian on the surface of the sphere takes the form [48]

$$
\mathcal{H}_{\text {surf }}^{0}=\frac{\Delta}{k_{F} R}\left[-i s_{2}\left(\partial_{\theta}+\frac{\cot \theta}{2}\right)+i \frac{s_{1}}{\sin \theta} \partial_{\phi}\right],
$$

and it gives rise to a spectrum analogous to that shown in Fig. 2(a), whose exact analytical form can be found in Ref. [48] (see also Appendix D). Due to the presence of the spin connection the Dirac equation on a curved surface has no zero eigenvalues.

It is clear that finite matrix elements between positiveand negative-energy states of the Hamiltonian (16) can arise only via the third Pauli matrix $s_{3}$ and that it is reasonable to conclude that in a curved geometry the vector potential has to couple to the spin connection. Alternatively, one may wonder how the Dirac Hamiltonian (5), describing the coupling of a Majorana spinor to a magnetic field that lies on the surface, changes when the surface acquires a finite curvature. On a flat Minkowski space with an in-plane magnetic field $\mathbf{B}=\boldsymbol{\nabla} \times \mathbf{A}$ pointing about the $x$ direction we can write Eq. (5) as

$$
\left(\gamma^{i}+\gamma^{0} a^{i}\right) \partial_{i} \psi=0
$$

where $a^{i}=\frac{e}{m v_{\Delta}}\left\langle A^{y}\right\rangle \delta_{y}^{i}$ and the only nonzero component of $A^{i}$ is fixed to lie in the plane, orthogonal to the magnetic field. The Majorana equation on the surface of the sphere is then obtained by introducing the covariant derivative

$$
\left(\gamma^{\mu}+\gamma^{0} a^{\mu}\right)\left(\partial_{\mu}+\Gamma_{\mu}\right) \psi=0
$$

where, analogous to $\gamma^{\mu}$, we have $a^{\mu}=a^{i} e_{i}^{\mu}$. Writing $a^{\phi}=$ $\frac{e}{m} A_{\phi}(R) /(R \sin \theta)$, with $A_{\phi}(R)=A(R) \sin \theta$, it follows that the Dirac Hamiltonian acquires a nontrivial term due to the coupling to the magnetic field

$$
\mathcal{H}_{\text {surf }}=\mathcal{H}_{\text {surf }}^{0}+\frac{\Delta}{k_{F} R} \frac{H}{H^{*}}\left(-i \partial_{\phi}-\frac{s_{3}}{2} \cos \theta\right) .
$$

We see that the spin connection introduces the relevant perturbation $s_{3} \cos \theta$. Introducing the half-integer total angular momentum eigenvalues $l=1 / 2,3 / 2, \ldots$, with projection along the polar axis $m=-l, \ldots, l$, the eigenvalues of the Dirac operator (16) read $\epsilon= \pm(l+1 / 2)$. Eigenfunctions can be written in terms of Jacobi polynomials and can be labeled by $l, m$, and $\sigma=\operatorname{sgn}(\lambda),\left|\Upsilon_{l m}^{\sigma}\right\rangle$. The spin connection then gives rise to the following matrix elements (see Appendix D):

$$
\begin{aligned}
\left\langle\Upsilon_{l m}^{+}\left|S_{3} \cos \theta\right| \Upsilon_{l^{\prime} m^{\prime}}^{-}\right\rangle= & i \delta_{m m^{\prime}} \frac{\sqrt{(l+1)^{2}-m^{2}}}{2(l+1)} \delta_{l^{\prime}, l+1} \\
& -i \delta_{m m^{\prime}} \frac{\sqrt{l^{2}-m^{2}}}{2 l} \delta_{l^{\prime}, l-1} .
\end{aligned}
$$

Upon identifying $(l, m)$ with $\left(j, j_{z}\right)$ we clearly see that the Majorana spin connection reproduces the matrix elements (13) that generate transitions between empty and occupied states differing by one unit of angular momentum, $l^{\prime}=l \pm 1$, and give rise to a finite response to the applied field.

\section{CONCLUSIONS}

We studied the coupling of a cone of Majorana modes localized at the surface of a class-DIII topological superconductor to an externally applied magnetic field. Upon taking into account Meissner screening and the associated supercurrent flow we found a Doppler-shift-mediated tilting of the Majorana cone that mirrors the entire spectrum modification. On a spherical geometry the surface curvature allows for a relevant coupling that is mediated by both the orbital and Zeeman terms and leads to the emergence of the Majorana spin connection. The effect acquires a universal character in 
finite-size systems that are topologically equivalent to the sphere. Our findings open the way to detection and manipulation of Majorana modes with time-dependent magnetic fields.

\section{ACKNOWLEDGMENTS}

The authors acknowledge very useful discussions with B. Bradlyn, F. de Juan, I. Grigorieva, and A. K. Geim. L.C. and F.G. acknowledge funding from the European Union's Seventh Framework Program (FP7/2007-2013) through the ERC Advanced Grant NOVGRAPHENE (Grant Agreement No. 290846); L.C. acknowledges funding from the Comunidad de Madrid through the grant MAD2D-CM, S2013/MIT-3007. F.G. acknowledges funding from the European Commission under the Graphene Flagship, Contract No. CNECTICT-604391.

\section{APPENDIX A: GEOMETRY-DEPENDENT SCREENING}

We consider the screening of an external field $H$ by typeII superconductors of spherical geometries. In general we have to solve a boundary problem; inside the boundary the vector potential $\mathbf{A}$ has a mass term $1 / \lambda^{2}$, and outside the boundary the mass term is zero. The vector potential can always be chosen to satisfy $\boldsymbol{\nabla} \cdot \mathbf{A}=0$. We can choose the vector potential to be purely azimuthal, $\mathbf{A}=\hat{\boldsymbol{\phi}} A_{\phi}(r)$. We then have to solve the following equation:

$$
\boldsymbol{\nabla} \times \boldsymbol{\nabla} \times \mathbf{A}=\left\{\begin{array}{cc}
-\frac{1}{\lambda^{2}} \mathbf{A} & r<R, \\
0 & r>R,
\end{array}\right.
$$

with the requirement that the vector potential at a sufficiently long distance from the sphere behaves as

$$
\lim _{r \gg R}\left(A_{r}, A_{\theta}, A_{\phi}\right) \rightarrow\left(0,0, \frac{H r}{2} \sin \theta\right) .
$$

We write $A_{\phi}=f(r) \sin \theta$ for $r \leqslant R$ and $A_{\phi}=g(r) \sin \theta$ for $r>R$. Continuity of the magnetic induction field $\mathbf{h}=\boldsymbol{\nabla} \times \mathbf{A}$ at $r=R$ requires that $f(R)=g(R)$ and $f^{\prime}(R)=g^{\prime}(R)$. For $r<R$ the function $f(r)$ has to satisfy

$$
\frac{f}{\lambda^{2}}=\frac{\partial^{2} f}{\partial r^{2}}+\frac{2}{r} \frac{\partial f}{\partial r}-\frac{2 f}{r^{2}},
$$

whose solution reads $A f(r)$, with

$$
f(r)=\operatorname{Im}\left[y_{-2}(i r / \lambda)\right]
$$

and $y_{v}(z)$ being a spherical Bessel function of the second kind. For $r>R$ the solution is

$$
g(r)=\frac{H r}{2}+\frac{C}{r^{2}}
$$

with $A$ and $C$ being real coefficients. By matching the solutions at $r=R$ we find

$$
\begin{aligned}
& A=H \frac{3 R}{2\left[2 f(R)+f^{\prime}(R) R\right]}, \\
& C=H \frac{R^{3}\left(f(R)-R f^{\prime}(R)\right)}{2\left[2 f(R)+f^{\prime}(R) R\right]} .
\end{aligned}
$$

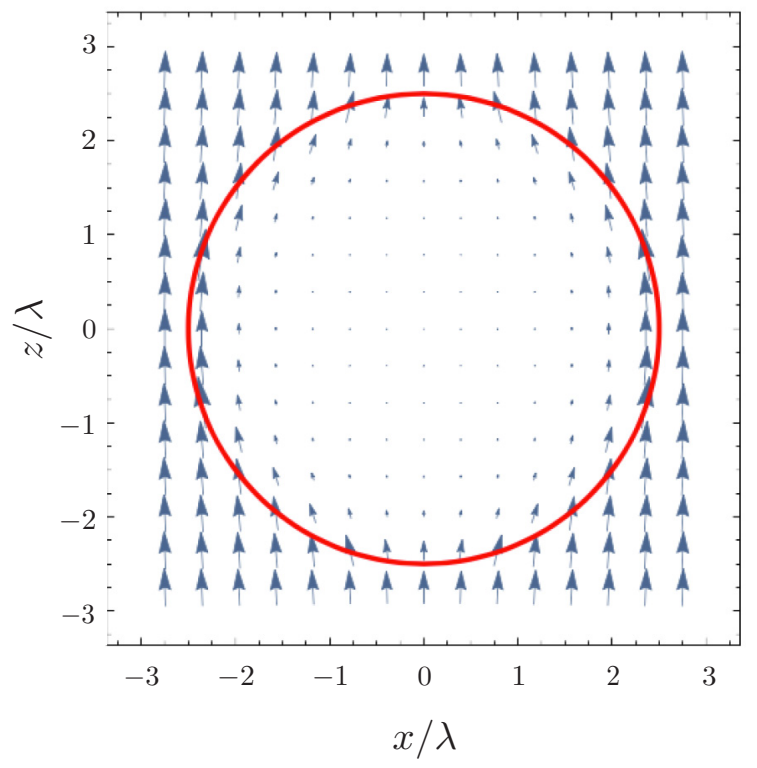

FIG. 3. Screened full-induction field $\mathbf{B}$ associated with the Meissner effect for $R=10 \lambda$.

The full induction field $\mathbf{B}=\boldsymbol{\nabla} \times \mathbf{A}$ then reads

$$
\mathbf{B}=\left\{\begin{array}{cc}
A \cos \theta \frac{2 f(r)}{r} \hat{\mathbf{r}}-A \sin \theta\left[\frac{f(r)}{r}+f^{\prime}(r)\right] \hat{\boldsymbol{\theta}} & r<R, \\
\cos \theta \frac{2 g(r)}{r} \hat{\mathbf{r}}-\sin \theta\left[\frac{g(r)}{r}+g^{\prime}(r)\right] \hat{\boldsymbol{\theta}} & r>R,
\end{array}\right.
$$

where $\hat{\mathbf{r}}$ and $\hat{\boldsymbol{\theta}}$ are unit vectors along the radial and polar directions, respectively. Approximating $-\operatorname{Im}\left[y_{-2}(i z)\right]=e^{z} /(2 z)$, it follows that the field for $r-R \rightarrow 0^{-}$reads

$$
\mathbf{B} \simeq \frac{3 H}{2}\left[\sin \theta \hat{\boldsymbol{\theta}}-\frac{\lambda}{2 R} \cos \theta \hat{\mathbf{r}}\right] .
$$

The full induction field is plotted in Fig. 3. We see that the field at the equator is parallel to the surface of the sphere and it has full strength of $\sim H$, whereas at the poles it is orthogonal to the surface and it has its strength reduced by a factor of $\lambda / R$ due to Meissner screening.

\section{APPENDIX B: BW STATE ON THE SPHERE}

We now study a purely finite size system on a sphere geometry. The BdG Hamiltonian is nothing more than the relativistic Dirac equation with a mass term that depends on momentum, $p^{2} / 2 m-\epsilon_{F}$, and the resulting second-order differential equation for a spherical boundary can be easily solved by recalling the relativistic central force problem. The subgap spectrum is found by imposing that the wave function is zero at the boundary, $\psi(R)=0$.

The problem can be reduced to a set of two coupled equations for the radial part of the wave function. It is possible to form a set of observables that commute with the Hamiltonian, $\hat{\mathbf{J}}^{2}, \hat{J}_{z}$ with quantum numbers $j=1 / 2,3 / 2, \ldots$ and $\left|j_{z}\right| \leqslant j$, and the operator $\hat{K}=\tau_{z}(\mathbf{s} \cdot \hat{\mathbf{L}}+\hbar)$, with eigenvalue $-\hbar \kappa$, where $\hat{\mathbf{L}}$ is the orbital angular momentum operator. The operator $\hat{K}$ plays an important role in that it divides the spectrum into positive- and negative-energy eigenstates, 
according to the sign of $\kappa= \pm(j+1 / 2)$. Thus, we denote the eigenstates of the Hamiltonian as $\left|\Psi_{j, j_{z}}^{\alpha}\right\rangle$, with $\alpha=\operatorname{sgn}(\kappa)$.

Separately, the electron and hole wave functions are eigenstates of $\hat{\mathbf{L}}^{2}$, with quantum numbers $l_{e}$ and $l_{h}$, respectively. The wave function is written as

$$
\Psi=\left(\begin{array}{l}
f(r) \mathcal{Y}_{j, l_{e}}^{j_{z}}(\theta, \phi) \\
g(r) \mathcal{Y}_{j, l_{h}}^{j_{z}}(\theta, \phi)
\end{array}\right)
$$

with $\mathcal{Y}_{j, l}^{j_{z}}(\theta, \phi)$ being the normalized spin-angular function. For a given eigenvalue $j=1 / 2,3 / 2, \ldots$, the operator $K$ has eigenvalues $-\hbar \kappa$, with $\kappa= \pm(j+1 / 2)$. The spin-angular function is eigenstates of $\hat{\mathbf{L}}$ with eigenvalue $l$, and it follows that for $\kappa=j+1 / 2$ we have that $l_{e}=j+1 / 2$ and $l_{h}=j-$ $1 / 2$, whereas for $\kappa=-j-1 / 2$ we have that $l_{e}=j-1 / 2$ and $l_{h}=j+1 / 2$. The relevant spin-angular wave function describing electron and hole components then reads

$$
\mathcal{Y}_{j, j \mp 1 / 2}^{j_{z}}=\left(\begin{array}{c} 
\pm \sqrt{\frac{j+1 / 2 \pm j_{z} \mp 1 / 2}{2 j+1 \mp 1}} Y_{j \mp 1 / 2}^{j_{z}-1 / 2} \\
\sqrt{\frac{j+1 / 2 \mp j_{z} \mp 1 / 2}{2 j+1 \mp 1}} Y_{j \mp 1 / 2}^{j_{z}+1 / 2}
\end{array}\right) .
$$

The problem is thus reduced to a coupled set of equations for the $f$ and $g$ functions. Defining $L_{\kappa}^{ \pm}=\left(\partial_{r} \mp \kappa / r\right)$, we can cast the problem in the form

$$
\left(\begin{array}{cc}
-\frac{\hbar^{2}}{2 m} D_{\kappa}-\mu-E & i \hbar v_{\Delta} L_{\kappa-1}^{+} \\
i \hbar v_{\Delta} L_{\kappa+1}^{-} & \mu+\frac{\hbar^{2}}{2 m} D_{\kappa-1}-E
\end{array}\right)\left(\begin{array}{l}
f \\
g
\end{array}\right)=0
$$

where we have defined $D_{\kappa}=\partial_{r}^{2}+(2 / r) \partial_{r}-\kappa(\kappa+1) / r^{2}$, which satisfies $D_{\kappa} \equiv L_{\kappa-1}^{+} L_{\kappa+1}^{-}$and $D_{\kappa-1}=L_{\kappa+1}^{-} L_{\kappa-1}^{+}$. Introducing the spherical Bessel functions of the first kind $j_{n}(x)=\sqrt{\frac{\pi}{2 x}} J_{n+1 / 2}(x)$, with $J_{n+1 / 2}$ being a Bessel function of the first kind and $n$ being an integer, and recalling the recurrence properties of the spherical Bessel functions

$$
\begin{aligned}
& L_{n+1}^{-} j_{n}(q r)=q j_{n-1}(q r), \\
& L_{n}^{+} j_{n}(q r)=-q j_{n+1}(q r),
\end{aligned}
$$

the eigenfunctions are given by spherical Bessel functions $f=A j_{\kappa}(k r)$ and $g=B j_{\kappa-1}(k r)$. We can compactly write the wave function as

$$
\Psi_{j, j_{z}}^{\alpha}=\left(\begin{array}{c}
A f_{l_{e}^{\alpha}}(r) \mathcal{Y}_{j, l_{e}^{\alpha}}^{j_{z}}(\theta, \phi) \\
B g_{l_{h}^{\alpha}}(r) \mathcal{Y}_{j, l_{h}^{\alpha}}^{j_{z}}(\theta, \phi)
\end{array}\right)
$$

The action of the operator $\hat{K}$ on these states is $\hat{K}\left|\Psi_{j, j_{z}}^{\alpha}\right\rangle=$ $-\alpha(j+1 / 2)\left|\Psi_{j, j_{z}}^{\alpha}\right\rangle$.

For the positive-energy eigenstates we have $\kappa=j+$ $1 / 2 \equiv \ell, l_{e}=\ell$, and $l_{h}=\ell-1$, so that we can write $f(r)=$ $A j_{\ell}(k r)$ and $g(r)=B j_{\ell-1}(k r)$ in terms of spherical Bessel functions. The wave vector $k$ is given by the solution of the determinantal equation

$$
\epsilon^{2}-\left(\epsilon_{F}-\frac{\hbar^{2} k^{2}}{2 m}\right)^{2}-\hbar^{2} v_{\Delta}^{2} k^{2}=0
$$

with $A=i v_{\Delta} k B\left[\epsilon+\epsilon_{F}-\hbar^{2} k^{2} /(2 m)\right]^{-1}$. For a given energy $\epsilon$ within the superconducting gap, $|\epsilon|<\Delta$, the possible values of $k$ for which the wave function vanishes at the origin $r=0$ are given by

$$
k_{ \pm}= \pm k_{F}+i \frac{\sqrt{2}}{\xi} \sqrt{1-\frac{\epsilon^{2}}{2 \Delta^{2}}} .
$$

The decaying length of these states in the bulk is the superconducting coherence length $\xi=\hbar v_{F} / \Delta$, with $v_{F}=\hbar k_{F} / m$, satisfying $k_{F} \xi=2 \epsilon_{F} / \Delta \gg 1$. By imposing the boundary condition $\psi(R)=0$ we find the spectrum of the problem. For large $k_{F} R$ the surface state spectrum below the gap is given by

$$
\epsilon_{j}^{+} \simeq \frac{\Delta}{k_{F} R}(j+1 / 2)
$$

Analogously, negative-energy eigenstates are found for the choice $\kappa=-j-1 / 2, l_{e}=\ell-1$, and $l_{h}=\ell$, so that we can write $f(r)=A j_{\ell-1}(k r)$ and $g(r)=B j_{\ell}(k r)$, with $\ell=$ $1,2, \ldots$, and find the subgap spectrum

$$
\epsilon_{j}^{-} \simeq-\frac{\Delta}{k_{F} R}(j+1 / 2) .
$$

For every $j$ we have $2 j+1$ degenerate states labeled by $j_{z}$. The spectrum is shown in Fig. 2(a) of the main text, and it is consistent with the generic spectrum of the Dirac equation on the surface of a sphere $[46,47]$. We see that the spectrum is gapped by an amount $2 \Delta / k_{F} R$ as a result of the finite size of the system and that the gap does not depend exponentially on the size of the system, contrary to the case of the planar geometry, only algebraically. It can be shown that the origin of the gapped spectrum is the spin connection that appears in the surface Hamiltonian on a curved surface.

\section{APPENDIX C: COUPLING TO THE MAGNETIC FIELD}

We now study the full coupling of the Majorana modes localized at the surface of a sphere of radius $R$ to the external field by first separately considering the orbital and Zeeman terms and then their joint action.

\section{Orbital term}

Neglecting the $\mathbf{A}^{2}$ term and choosing the gauge $\mathbf{A}=$ $\hat{\phi} A_{\phi}(\mathbf{r})$, the magnetic field couples via the term

$$
\mathcal{H}_{\mathbf{p} \cdot \mathbf{A}}=\frac{e}{m} p_{\phi} A_{\phi}(\mathbf{r}),
$$

with $p_{\phi}=-\frac{i \hbar}{r \sin \theta} \partial_{\phi}$. The vector potential can be chosen to have the form $A_{\phi}(\mathbf{r})=A(r) \sin \theta$. Due to the spin-triplet pairing the azimuthal momentum $p_{\phi}$ is not a constant of motion. We write the $z$ component of the angular momentum as $L_{z} \equiv-i \hbar \partial_{\phi}=\hat{J}_{z}-\hbar s_{z} / 2$, so that we can separate $\mathcal{H}_{\mathbf{p} \cdot \mathbf{A}}$ into a term proportional to $\hat{J}_{z}$ and a term proportional to $s_{z}$,

$$
\mathcal{H}_{\mathbf{p} \cdot \mathbf{A}}=\frac{e}{m} \frac{A_{\phi}(\mathbf{r})}{r \sin \theta}\left(\hat{J}_{z}-\frac{\hbar}{2} s_{z}\right)
$$

For large $j_{z}$ we can neglect the term proportional to $s_{z}$. In the limit $R \gg \lambda \gg \xi$ we can approximate the matrix elements of the term proportional to $J_{z}$ as

$$
\left\langle\Psi_{j, j_{z}}^{\alpha}\left|\frac{e}{m} \frac{A(r)}{r} J_{z}\right| \Psi_{j, j_{z}}^{\alpha}\right\rangle=-j_{z} \frac{\hbar^{2}}{2 m} \frac{2 \pi H}{\Phi_{0}} \frac{\lambda}{R} .
$$


We thus see that the magnetic field produces a tilting of the spectrum, in analogy with the case of the planar and cylindrical surface. The modified spectrum at large $j_{z}$ is given by

$$
\epsilon_{j, j_{z}}^{ \pm}= \pm \frac{\Delta}{k_{F} R} j-j_{z} \frac{\hbar^{2}}{2 m} \frac{2 \pi H}{\Phi_{0}} \frac{\lambda}{R} .
$$

The energy of the states with $j_{z}>0$ is lowered, while the energy of the states with $j_{z}<0$ is raised. The threshold field is then given by

$$
H^{*}=\frac{\Phi_{0}}{2 \pi} \frac{2}{\lambda \xi},
$$

which is consistent with the results of planar and cylindrical geometries.

For small values of $j_{z}$ the contribution proportional to $s_{z}$ becomes important. The spin operator $s_{z}$ does not commute with the operator $\hat{K}=\tau_{z}(\mathbf{s} \cdot \hat{\mathbf{L}}+\hbar)$, so that it will, in general, couple states with positive energy with states with negative energy. Furthermore, since $s_{z}$ is even under parity, it can couple only states differing by an odd number of angular momentum quanta. In particular, we find that $s_{z}$ has finite matrix elements between states of positive energy and quantum numbers $\left(j, j_{z}\right)$ and states of negative energy with quantum numbers $\left(j \pm 1, j_{z}\right)$.

Assuming that in the limit $\xi / \lambda \ll 1$ the field does not substantially vary in the region $R-\xi<r<R$, where the radial integral has its largest contribution due to the localization of the Majorana wave function, it can be taken outside the integral, and the relevant matrix elements amount to

$$
\left\langle\Psi_{j, j_{z}}^{+}\left|s_{z}\right| \Psi_{j \pm 1, j_{z}}^{-}\right\rangle=-\frac{\sqrt{(j+1 / 2 \pm 1 / 2)^{2}-j_{z}^{2}}}{j+1 / 2 \pm 1 / 2},
$$

providing a finite coupling between positive- and negativeenergy states that increases the gap in the spectrum.

\section{Zeeman term}

We now consider the action of the Zeeman term. In the spherical geometry its surface contribution reads

$$
H_{Z}=g \mu_{B} \frac{3 H}{2}\left[\sin \theta \hat{\boldsymbol{\theta}}-\frac{\lambda}{2 R} \cos \theta \hat{\mathbf{r}}\right] \cdot \mathbf{s} .
$$

Making use of the identity

$$
\hat{\mathbf{r}} \cdot \mathbf{s}\left|\mathcal{Y}_{j, j \pm 1 / 2}^{j_{z}}\right\rangle=-\left|\mathcal{Y}_{j, j \mp 1 / 2}^{j_{z}}\right\rangle,
$$

together with $\{\hat{\boldsymbol{\theta}} \cdot \mathbf{s}, \hat{\mathbf{r}} \cdot \mathbf{s}\}=0$, we see that the matrix element of the polar spin component $\hat{\boldsymbol{\theta}} \cdot \mathbf{s}$ is approximately zero. More precisely, one has

$$
\begin{aligned}
& \left\langle\Psi_{j, j_{z}}^{+}|\sin \theta \hat{\boldsymbol{\theta}} \cdot \mathbf{s}| \Psi_{j^{\prime} \cdot j_{z}^{\prime}}^{-}\right\rangle \\
& =\delta_{j_{z}, j_{z}^{\prime}} \int_{0}^{R} d r r^{2}\left[f_{j}^{+}(r)^{*} f_{j^{\prime}}^{-}(r)-g_{j}^{+}(r)^{*} g_{j^{\prime}}^{-}(r)\right] \\
& \quad \times\left[c_{j, j_{z}}^{0} \delta_{j, j^{\prime}}+c_{j, j_{z}}^{+} \delta_{j^{\prime}, j+2}+c_{j, j_{z}}^{-} \delta_{j^{\prime}, j-2}\right] .
\end{aligned}
$$

In fact, although the angular integral has nonzero matrix elements for $j^{\prime}=j$ and $j^{\prime}=j \pm 2$, the radial integral is strongly suppressed. It follows that this component has no effect on the Majorana modes, which are found to be insensitive to in-plane fields.

Close to the poles, the field points about the radial direction, which is orthogonal to the surface and fully couples to the Majorana modes. Its magnitude is weakened by a factor $\lambda / R$, which makes it comparable to the orbital term proportional to $s_{z}$. Furthermore, the angular matrix elements of the relevant part of the orbital and Zeeman terms are the same, and an assessment of their relative sign and magnitude becomes crucial.

\section{Orbital and Zeeman terms}

The full coupling with the magnetic field is written as

$$
H_{B}=\frac{e}{m} A_{\phi}(\mathbf{r}) p_{\phi}+g^{*} \mu_{B} \mathbf{B}(\mathbf{r}) \cdot \mathbf{s},
$$

where $g^{*}=g / 2$ is the ratio between the material $g$ factor and that of the bare electron. Recalling that $A_{\phi}(\mathbf{r})=$ $A(r) \sin \theta, \quad p_{\phi}=-i \hbar \partial_{r} /(r \sin \theta), \quad-i \partial_{r}=j_{z}-s_{z} / 2, \quad$ and $\mu_{B}=e \hbar /(2 m)$, we have

$$
\begin{aligned}
H_{B}= & \frac{e \hbar A(r)}{m r} j_{z}-\mu_{B}\left(\frac{A(r)}{r} s_{z}-g^{*} \mathbf{B}(\mathbf{r}) \cdot \mathbf{s}\right) \\
= & \frac{e \hbar A(r)}{m r} j_{z}-g^{*} \mu_{B} \frac{A(r)}{r}\left(1+\frac{A^{\prime}(r) r}{A(r)}\right) \sin \theta \hat{\boldsymbol{\theta}} \cdot \mathbf{s} \\
& -\mu_{B} \frac{A(r)}{r}\left(s_{z}-2 g^{*} \cos \theta \hat{\mathbf{r}} \cdot \mathbf{s}\right) .
\end{aligned}
$$

In the first line of the second equation we recognize the tilting term and the parallel-to-the-surface polar term. The relevant part of the magnetic field that has nonvanishing matrix elements between positive- and negative-energy states is given by the third term. We are then left with the calculation of the matrix elements of

$$
M_{j, j^{\prime}}=-\mu_{B} \frac{3 H \lambda}{2 R}\left\langle\Psi_{j, j_{z}}^{+}\left|s_{z}-2 g^{*} \cos \theta \hat{\mathbf{r}} \cdot \mathbf{s}\right| \Psi_{j^{\prime}, j_{z}}^{-}\right\rangle .
$$

Both the terms $s_{z}$ and $\cos \theta \hat{\mathbf{r}} \cdot \mathbf{s}$ are even under parity, so that $j$ and $j^{\prime}$ have to differ by a unit of angular momentum. Defining

$$
\begin{aligned}
u_{j, j^{\prime}} & =\int_{0}^{R} d r r^{2} f_{j}^{+}(r)^{*} f_{j^{\prime}}^{-}(r), \\
v_{j, j^{\prime}} & =\int_{0}^{R} d r r^{2} g_{j}^{+}(r)^{*} g_{j^{\prime}}^{-}(r),
\end{aligned}
$$

where we extended the radial integral to the entire $r<R$ region, we have

$$
\begin{aligned}
\left\langle\Psi_{j, j_{z}}^{+}\left|s_{z}\right| \Psi_{j^{\prime}, j_{z}}^{-}\right\rangle & =u_{j, j^{\prime}} \int d \Omega\left(\mathcal{Y}_{j, j+1 / 2}^{j_{z}}\right)^{*} s_{z} \mathcal{Y}_{j^{\prime}, j^{\prime}-1 / 2}^{j_{z}}+v_{j, j^{\prime}} \int d \Omega\left(\mathcal{Y}_{j, j-1 / 2}^{j_{z}}\right)^{*} s_{z} \mathcal{Y}_{j^{\prime}, j^{\prime}+1 / 2}^{j_{z}} \\
& =-u_{j, j^{\prime}} \frac{\sqrt{(j+1)^{2}-j_{z}^{2}}}{j+1} \delta_{j^{\prime}, j+1}-v_{j, j^{\prime}} \frac{\sqrt{\left(j^{\prime}+1\right)^{2}-j_{z}^{2}}}{j^{\prime}+1} \delta_{j^{\prime}+1, j},
\end{aligned}
$$




$$
\begin{aligned}
\left\langle\Psi_{j, j_{z}}^{+}|\cos \theta \hat{\boldsymbol{r}} \cdot \mathbf{s}| \Psi_{j^{\prime}, j_{z}^{\prime}}^{-}\right\rangle & =-\left(u_{j, j^{\prime}}+v_{j, j}\right) \int d \Omega \cos \theta\left(\mathcal{Y}_{j, j+1 / 2}^{j_{z}}\right)^{*} \mathcal{Y}_{j^{\prime}, j^{\prime}+1 / 2}^{j_{z}} \\
& =-\frac{u_{j, j^{\prime}}+v_{j, j^{\prime}}}{2}\left[\frac{\sqrt{(j+1)^{2}-j_{z}^{2}}}{j+1} \delta_{j^{\prime}, j+1}+\frac{\sqrt{\left(j^{\prime}+1\right)^{2}-j_{z}^{2}}}{j^{\prime}+1} \delta_{j^{\prime}+1, j}\right] .
\end{aligned}
$$

Approximating $u_{j, j-1} \simeq v_{j, j+1} \simeq 1 / 2$, we see that the orbital term is completely canceled by part of the Zeeman term and the relevant matrix elements amount to

$$
\left\langle\Psi_{j, j_{z}}\left|H_{B}\right| \Psi_{j \pm 1, j_{z}}\right\rangle=\tilde{g} \mu_{B} \frac{3 H \lambda}{2 R} \frac{\sqrt{(j+1 / 2 \pm 1 / 2)^{2}-j_{z}^{2}}}{j+1 / 2 \pm 1 / 2},
$$

with $\tilde{g}=g^{*}-1 / 2$. It follows that the two terms do not cancel completely and that a relevant perturbation results from the combined action of the two terms, providing a relevant perturbation to the Majorana modes.

\section{APPENDIX D: EIGENFUNCTIONS OF THE SURFACE DIRAC OPERATOR}

Here, we provide the eigenfunctions of the 2D Dirac operator of the surface of the sphere. For simplicity we set the velocity to $1, v_{\Delta}=1$. The Dirac operator is given by Eq. (13) in the main text, and we repeat it here for convenience. Redefining the Pauli matrices appearing in the main text as $\left(s_{x}, s_{y}, s_{z}\right) \rightarrow\left(-\sigma_{y}, \sigma_{x}, \sigma_{z}\right)$, the Dirac operator reads

$$
H=-i \sigma_{x}\left(\partial_{\theta}+\frac{\cot \theta}{2}\right)-i \frac{\sigma_{y}}{\sin \theta} \partial_{\phi} .
$$

The eigenfunctions are provided in Ref. [48], and we refer to that reference for details and conventions. The Dirac operator on the sphere $S^{2}$ is invariant under transformation of the $\mathrm{SU}(2)$ group, whose generators are given by

$$
\begin{gathered}
\hat{L}_{z}=-i \partial_{\phi}, \\
\hat{L}_{+}=e^{i \phi}\left(\partial_{\theta}+i \cot \theta \partial_{\phi}+\frac{\sigma_{z}}{2 \sin \theta}\right), \\
\hat{L}_{-}=e^{i \phi}\left(\partial_{\theta}-i \cot \theta \partial_{\phi}-\frac{\sigma_{z}}{2 \sin \theta}\right),
\end{gathered}
$$

which satisfy standard commutation rules of the $\mathrm{SU}(2)$ algebra, $\left[\hat{L}_{z}, \hat{L}_{+}\right]=\hat{L}_{+},\left[\hat{L}_{z}, \hat{L}_{-}\right]=-\hat{L}_{-},\left[\hat{L}_{+}, \hat{L}_{-}\right]=2 \hat{L}_{z}$. The Casimir operator $\hat{L}^{2}$ takes eigenvalues $l(l+1)$, with half integer $l=1 / 2,3 / 2, \ldots$. Eigenstates can be labeled by the quantum numbers $l$ and $m$, with $m=-l, \ldots, l$ being the eigenvalue of $\hat{L}_{z}$. The eigenfunctions of the Dirac operator are specified by integer eigenvalues

$$
\lambda= \pm(l+1 / 2) .
$$

We then introduce integers $l_{ \pm}=l \pm 1 / 2$ and $m_{ \pm}=m \pm 1 / 2$ and write the eigenfunctions as $(x=\cos \theta)$

$$
\begin{aligned}
\Upsilon_{l m}^{ \pm}(x, \phi)= & i_{+}^{l}(-1)^{\frac{1}{2}\left(m_{-}+\left|m_{-}\right|\right)} \frac{\sqrt{(l+m) !(l-m) !}}{2^{|m|+1 / 2} \Gamma\left(l_{+}\right)} \frac{e^{i m \phi}}{\sqrt{2 \pi}} \\
& \times\left(\begin{array}{c}
\sqrt{\rho^{\left(\left|m_{-}\right|,\left|m_{+}\right|\right)}(x)} P_{l-|m|}^{\left(\left|m_{-}\right|,\left|m_{+}\right|\right)}(x) \\
\operatorname{sgn}(m \lambda) \sqrt{\rho^{\left(\left|m_{+}\right|,\left|m_{-}\right|\right)}(x)} P_{l-|m|}^{\left(\left|m_{+}\right|,\left|m_{-}\right|\right)}(x)
\end{array}\right),
\end{aligned}
$$

where $\rho^{(\alpha, \beta)}(x)=(1-x)^{\alpha}(1+x)^{\beta}$ is the weight function for the Jacobi polynomials $P^{(\alpha, \beta)}$ [49]. The eigenstates are normalized as $\left\langle\Upsilon_{l m}^{\sigma} \mid \Upsilon_{l^{\prime} m^{\prime}}^{\sigma^{\prime}}\right\rangle=\delta_{\sigma, \sigma^{\prime}} \delta_{l, l^{\prime}} \delta_{m, m^{\prime}}$.

\section{Matrix elements of the spin connection}

We now have all the elements to calculate the matrix elements of the spin connection

$$
\Gamma_{\phi}=-i \frac{\sigma_{z}}{2} \cos \theta
$$

The matrix elements can be obtained via application of the fundamental identity [49]

$$
\begin{aligned}
& 2(n+1)(n+\alpha+\beta+1)(2 n+\alpha+\beta) P_{n+1}^{(\alpha, \beta)}(x) \\
&= {\left[(2 n+\alpha+\beta)(2 n+\alpha+\beta+2) x+\alpha^{2}-\beta^{2}\right] } \\
& \times(2 n+\alpha+\beta+1) P_{n}^{(\alpha, \beta)}(x) \\
&-2(n+\alpha)(n+\beta)(2 n+\alpha+\beta+2) P_{n-1}^{(\alpha, \beta)}(x) .
\end{aligned}
$$

Together with the matrix elements provided in the main text, it is possible to show that

$$
\left\langle\Upsilon_{l m}^{\sigma}\left|x \sigma_{z}\right| \Upsilon_{l^{\prime} m^{\prime}}^{\sigma}\right\rangle=\delta_{m m^{\prime}} \delta_{l^{\prime}, l} \frac{m}{2 l(l+1)}
$$

which slightly corrects the tilting term.
[1] E. Majorana, Nuovo Cimento 14, 171 (2008).

[2] J. Alicea, Rep. Prog. Phys. 75, 076501 (2012).

[3] D. A. Ivanov, Phys. Rev. Lett. 86, 268 (2001).

[4] A. Y. Kitaev, Phys. Usp. 44, 131 (2001).

[5] V. Mourik, K. Zuo, S. M. Frolov, S. R. Plissard, E. P. A. M. Bakkers, and L. P. Kouwenhoven, Science 336, 1003 (2012).

[6] X.-L. Qi and S.-C. Zhang, Rev. Mod. Phys. 83, 1057 (2011).

[7] Y. Ando and L. Fu, Annu. Rev. Condens. Matter Phys. 6, 361 (2015).
[8] B. A. Bernevig, Topological Insulators and Topological Superconductors (Princeton University Press, Princeton, NJ, 2013).

[9] M. Z. Hasan and C. L. Kane, Rev. Mod. Phys. 82, 3045 (2010).

[10] C.-K. Chiu, J. C. Y. Teo, A. P. Schnyder, and S. Ryu, Rev. Mod. Phys. 88, 035005 (2016).

[11] S. Tewari, S. Das Sarma, C. Nayak, C. Zhang, and P. Zoller, Phys. Rev. Lett. 98, 010506 (2007).

[12] C. Nayak, S. H. Simon, A. Stern, M. Freedman, and S. Das Sarma, Rev. Mod. Phys. 80, 1083 (2008). 
[13] A. Altland and M. R. Zirnbauer, Phys. Rev. B 55, 1142 (1997).

[14] A. P. Schnyder, S. Ryu, A. Furusaki, and A. W. W. Ludwig, Phys. Rev. B 78, 195125 (2008).

[15] A. Kitaev, in Advances in Theoretical Physics: Landau Memorial Conference, AIP Conf. Proc. No. 1134 (AIP, New York, 2009), p. 22.

[16] F. Zhang, C. L. Kane, and E. J. Mele, Phys. Rev. Lett. 111, 056403 (2013).

[17] S. Ryu, A. P. Schnyder, A. Furusaki, and A. W. W. Ludwig, New J. Phys. 12, 065010 (2010).

[18] G. E. Volovik, The Universe in a Helium Droplet (Oxford University Press, New York, 2003).

[19] X.-L. Qi, T. L. Hughes, S. Raghu, and S.-C. Zhang, Phys. Rev. Lett. 102, 187001 (2009).

[20] X.-L. Qi, T. L. Hughes, and S.-C. Zhang, Phys. Rev. B 81, 134508 (2010).

[21] H. Zhang, C.-X. Liu, X.-L. Qi, X. Dai, Z. Fang, and S.-C. Zhang, Nat. Phys. 5, 438 (2009).

[22] C. Fang, M. J. Gilbert, and B. A. Bernevig, Phys. Rev. B 86, 115112 (2012).

[23] L. Fu, C. L. Kane, and E. J. Mele, Phys. Rev. Lett. 98, 106803 (2007).

[24] Y. L. Chen, J. G. Analytis, J.-H. Chu, Z. K. Liu, S.-K. Mo, X. L. Qi, H. J. Zhang, D. H. Lu, X. Dai, Z. Fang, S. C. Zhang, I. R. Fisher, Z. Hussain, and Z.-X. Shen, Science 325, 178 (2009).

[25] L. Fu and E. Berg, Phys. Rev. Lett. 105, 097001 (2010).

[26] Y. S. Hor, A. J. Williams, J. G. Checkelsky, P. Roushan, J. Seo, Q. Xu, H. W. Zandbergen, A. Yazdani, N. P. Ong, and R. J. Cava, Phys. Rev. Lett. 104, 057001 (2010).

[27] M. Kriener, K. Segawa, Z. Ren, S. Sasaki, and Y. Ando, Phys. Rev. Lett. 106, 127004 (2011).

[28] H. Peng, D. De, B. Lv, F. Wei, and C.-W. Chu, Phys. Rev. B 88, 024515 (2013).

[29] S. Sasaki, M. Kriener, K. Segawa, K. Yada, Y. Tanaka, M. Sato, and Y. Ando, Phys. Rev. Lett. 107, 217001 (2011).
[30] Z. Wang, A. A. Taskin, T. Frölich, M. Braden, and Y. Ando, Chem. Mater. 28, 779 (2016).

[31] L. A. Wray, S.-Y. Xu, Y. Xia, Y. S. Hor, D. Qian, A. V. Fedorov, H. Lin, A. Bansil, R. J. Cava, and M. Z. Hasan, Nat. Phys. 6, 855 (2010).

[32] J. W. F. Venderbos, V. Kozii, and L. Fu, Phys. Rev. B 94, 180504 (2016).

[33] C. Beenakker, Annu. Rev. Condens. Matter Phys. 4, 113 (2013).

[34] R. Aguado, Riv. Nuovo Cimento 40, 523 (2017).

[35] S. B. Chung and S.-C. Zhang, Phys. Rev. Lett. 103, 235301 (2009).

[36] A. A. Soluyanov, D. Gresch, Z. Wang, Q. Wu, M. Troyer, X. Dai, and B. A. Bernevig, Nature (London) 527, 495 (2015).

[37] B. Bradlyn, J. Cano, Z. Wang, M. G. Vergniory, C. Felser, R. J. Cava, and B. A. Bernevig, Science 353, aaf5037 (2016).

[38] R. Balian and N. R. Werthamer, Phys. Rev. 131, 1553 (1963).

[39] A. A. Abrikosov, L. P. Gor'kov, and I. E. Dzialoshinskii, Methods of Quantum Field Theory in Statistical Physics (Dover, New York, 1963).

[40] M. Sigrist and K. Ueda, Rev. Mod. Phys. 63, 239 (1991).

[41] P. G. de Gennes, Superconductivity of Metals and Alloys (Addison-Wesley, New York, 1966), p. 153.

[42] T. H. Hsieh and L. Fu, Phys. Rev. Lett. 108, 107005 (2012).

[43] L. Chirolli and F. Guinea, arXiv:1802.09204.

[44] L. Chirolli, F. de Juan, and F. Guinea, Phys. Rev. B 95, 201110 (2017).

[45] N. Nakahara, Geometry, Topology and Physics (Hilger, London, 1990).

[46] J. González, F. Guinea, and M. A. H. Vozmediano, Phys. Rev. Lett. 69, 172 (1992).

[47] V. Parente, P. Lucignano, P. Vitale, A. Tagliacozzo, and F. Guinea, Phys. Rev. B 83, 075424 (2011).

[48] A. A. Abrikosov, Jr., arXiv:hep-th/0212134.

[49] I. S. Gradshteyn and I. M. Ryzhik, Tables of Integrals, Series, and Products (Academic Press, Cambridge, Massachusetts, 2007). 